\title{
Using Double Helix Relationships to Understand and Change Informing Syste ms
}

\section{Hans-Erik Nissen \\ Department of Information and Computer Science University of Lund, Sweden}

\author{
hans.nissen@telia.com
}

\begin{abstract}
The paper opens by generalizing the concept of 'informing science'. It then introduces some meta-scientific perspectives and a discussion of a metaphor that has considerable explanatory power. Two main schools of metascience are presented and contrasted. The difference between treating invariances in natural sciences and in social and cultural sciences is discussed. The double helix is introduced as a generic metaphor to highlight important distinctions. Highlighting new distinctions in this way can help to avoid simply assimilating them into already familiar distinctions. The paper also discusses how some metascientific perspectives and the transdisciplinary generalized concept of informing science can be seen as related. Finally, the paper argues that computerized models never keep up with continuously changing situations. However, people always have to handle the full variety of situations, including those not foreseen during requirements engineering. To address this, the paper suggests balancing requirements engineering with model transparency engineering
\end{abstract}

Keywords: cognition versus recognition, double helix metaphor, hermeneutics-dialectics, information systems design, information systems

Material published as part of this publication, either on-line or in print, is copyrighted by the Informing Science Institute. Permission to make digital or paper copy of part or all of these works for personal or classroom use is granted without fee provided that the copies are not made or distributed forprofit or commercial advantage AND that copies 1) bear this notice in full and 2) give the full citation on the first page. It is permissible to abstract these works so long as credit is given. To copy in all other cases or to republish or to post on a server or to redistribute to lists requires specific permission and payment of a fee. Contact 1HPublisher@InformingScience.org to request redistribution permission. 
use, informing science, language, languaging, logical-empiricism, metascience, model transparency engineering

\section{Introduction}

In his 1999 paper Cohen introduces Informing Science as a transdiscipline bridging among "a number of disparate fields that share some common goals". He defines the fields, which form the discipline of Informing Science, in the following way:

The fields that comprise the discipline of Informing Science provide their clientele with information in a form, format, and schedule that maximizes its effectiveness (Cohen, 1999).

In this paper I shall demarcate the field I call informing science in a slightly different - more general -manner as follows:

The fields that comprise the discipline of Informing Science provide their clientele with data and meta-data in a form, format, and schedule that the clientele find ever more valuable.

First, I have substituted the word "data" instead of "information". My reason is to safeguard an autonomous right of interpretation to receivers of messages. Second, I have avoided the concept of 'maximization'. This concept lacks precise meaning outside the realm of specified formal (mathematical) models. Third, I have introduced the clientele to evaluate data with which they are furnished in their work or life situations. This reflects the fact that different groups of clientele apply different sets of value criteria. It also acknowledges that a particular group of clientele may change its set of value criteria over time. Fourth, I have accommodated dynamic variability by the phrase "ever more". As clientele values and situations develop over time, so will data and their forms, formats, and schedules. Finally, the reference to meta-data opens the way for including disciplines studying how human expressiveness evolves.

My more general demarcation does notinvalidate Cohen's original definition. It remains valid provided a set of restrictions are explicitly introduced. Generalizing the discipline of informing science implies a corresponding broadening of the concept of an 'informing system'. Both the original and the generalized characterization of informing science focus on clientele. The latter emphasizes the clientele as interpreters by substituting "data and meta-data" for "information". Today it 
might be argued it is not only people who can interpret. Artificial agents can do so, too. Be this as it may. I will delimit the concept of an 'informing system' in the following way. Its effective use has to include parts of closed cause-effect chains that are not confined to artifacts. In the general demarcation what constitutes effective use remains open for the clientele to decide. (In the subsection on "Generalized informing systems" this point will be discussed somewhat more.)

Because it connotes subsumption of multiple 'sciences' (i.e., fields), the very idea of a transdiscipline requires an explanatory approach at the level of metascience. Against this background this paper aims to present some metascientific perspectives and a generic metaphor. The metaphor is intended to acknowledge and emphasize a distinction important for practitioners and researchers. This is the distinction between living and acting in a concrete here-and-now versus reflecting upon what has passed or may come. Reflecting is always done using a net of concepts mediated, mainly by language, in a society. The net of concepts used focuses and constrains explicit explanations given and predictions made. Such a net of concepts is generally taken for granted by the members of some subsociety. The distinction helps one to understand that a single such net of concepts cannot be adequate to explain or predict all human living and acting. Giddens (1984, p. 7) discusses a somewhat similar distinction between what he calls "discursive consciousness" and "practical consciousness".

A focus on use entails a focus on people, their societies, and cultures and how they change them over time. In their attempts to make sense of their lives, societies, and cultures, people use languages to discuss whether to perpetuate or change their ways of living. To some extent such discussions entail debates about designing new artifacts to improve human life. These debates can be seen as occurring within two partially overlapping arenas. One can be called an arena of craftsmanship and engineering and the other a scientific arena. There currently exists a rather common belief that the scientific arena always leads and paves the way for engineering progress. More fruitfully (and accurately) these two arenas can be seen instead as mutually stimulating each other. Moreover, many products of scientists do not seem to have any impact on human practice. In particular, this seems the case when it comes to findings from fields within the social sciences. This can depend upon the tradition within which any particular study is undertaken. 
In the next section I will discuss the metascience of different scientific schools and some fundamental differences in social science research. I will end with a subsection relating metascientific ideas to studying and meeting demands for changes in informing systems. In the following main section I will discuss a number of views of using a double helix as a generic metaphor. Following that, the next section will go somewhat deeper into how informing science and metascience can be seen as related. The paper will finally present a few conclusions.

\section{Scientific Traditions and Social Science Studies}

\section{Two Schools of Metascience}

In the English speaking world the word "science" generally refers only to natural sciences. In this paper, following Radnitzky (1970), I will use words as "science and scientific" in a broader sense encompassing social and cultural sciences as well. Radnitzky (1970, Vol. I, p. 6) uses the term "metascience" somewhat differently from the expression "philosophy of science". He perceives metascience as a scientific discipline that accumulates knowledge about the scientific enterprise, checks this knowledge and organizes it into knowledge systems. (See also "metascience" in the Glossary accompanying this monograph.)

In both its broad and in its narrow sense scientific knowledge is necessarily both specialized and fragmentary. People who want to take advantage of scientific knowledge in some practical situation need to synthesize findings from many disciplines. This they will be better equipped to do, if they are aware of the different scientific traditions of various researchers. Informing science researchers and practitioners want to work within a transdiscipline. For them an awareness of different schools of metascience can help them communicate across disciplinary boundaries.

Radnitzky (1970) distinguishes between Anglo-Saxon or logical-empirical (LE) and Continental or bermeneutic-dialectic (HD) schools of metascience. . According to Radnitzky these should be perceived as "two bighly-stylized ideal types that might be used to polarize the existing types of "philosophical enterprises" (Vol. I, p. 15.) Both these main traditions are comprised of a number of sub-schools. Within the fields of information and computer science the LE school has dominated. 
For at least two decades within information science a number of studies applying HD methods of research have appeared. This monograph addresses how ideas from philosophies behind HD traditions could support informing science research and practice. I shall accordingly focus on perspectives from HD traditions. However, this must not be interpreted to imply that methods from HD traditions should dominate over methods from LE traditions. As Radnitzkty (1970, Vol. II, pp. 6566) argues the two traditions should be seen as complementary. HD traditions, to be self-sufficient, would presuppose that man is fully transparent to himself. This is simply not the case. Taking LE as the only legitimate form of scientific study would reduce all human sciences to behavioral science. Such a reductive ideal of social science must, if consequently adhered to, accept a society divided into manipulators and manipulated.

The following differences between Radnitzky's two main schools of metascience are important.

LE schools presume:

- to state objectively true facts about an observer independent reality,

- that historical contexts are irrelevant to data collected, (mainly synchronic observations)

- that only values intrinsic to science shall guide research,

- that theory and practice can be strictly separated,

- that language used should be extensional and denotational,

- that they form the bases for technologies and emancipate man from nature.

HD schools presume:

- to state intersubjectively sharable relations between observers and a territory observed,

- that historical contexts are relevant to data collected, (diachronic observations important)

- that both science intrinsic and extrinsic values shall guide research, 
- that theory and practice are mutually related,

- that language used should be

mainly intentional and connotational,

- that they support understanding, mediation of traditions, and emancipation by improved self-understanding.

Researchers of these two meta-scientific schools perceive their work tasks differently. (Here I distinguish them in a similar way to what Radnitzky, 1970, Vol. I, p. 15-16, does with two types of philosophers.) The work of LE researchers must be respectable. They have to adhere to the methods of validating scientific findings according to their tradition. Moreover, as theory and practice are seen as strictly separate they cannot be held accountable for what their findings lead to. They must remain as clarifying on-lookers and sharpeners of (LE) scientific tools. (This reflects the principles of a LE position. Many researchers working within LE traditions are still concerned about where their findings may lead.) In contrast $\mathrm{HD}$ researchers are engaged in their work. It is essential for them, both socially and personally, to mediate between their theoretical findings and the practice of life. For them the practice of life constitutes the basic human problem.

To increase what we, as humans, know and can build our future societies and lives upon, both these types of researchers are needed. However, they have different strengths with respect to describing and explaining different kinds of subject matter. Working according to LE research traditions is effective for studying inanimate subjects - e.g., in a field such as physics. Working according to HD research traditions shows its strengths when the subject matter includes people - i.e. subjects that involve living human beings forming groups and societies.

A critique of the LE methods of verification is foreshadowed in Giambattista Vico's work from the beginning of the 18th century. He contested Descartes' method of verification (through logical deduction from axioms derived from observation) as universally applicable (Stanford Encyclopedia of Philosophy, 2007, section 3.). He did not argue that the Cartesian method was irrelevant. His position was that other methods were needed for studies that extended to civil societies. These methods had to account for history and language including rhetoric and use of metaphor. According to Vico, knowledge in human sciences is 
verified not through logic but through creation. This is known as his principle of verum factum.

\section{Galtung's View on Social Science Studies}

Galtung's ideas about social science studies, stressing potential worlds, are reminiscent of Vico's views on creation and invention. Galtung (1977, p. 57) distinguishes three types of sentences in research: (a) data sentences, (b) theory sentences, and (c) value sentences (external to science). Galtung wrote at a time before it was common to acknowledge the importance of values external to science in information systems studies. Moreover, he thereby exposed the connection between methodology and ideology. He also introduced the important distinction, discussed below, between invariance seeking and invariance breaking.

Traditional empirical science, according to Galtung (1977, p. 51-56), only compares data sentences and theory sentences. If they show dissonance, data sentences are perceived as stronger than theory sentences. A dissonant theory sentence (hypothesis) is discarded as false or revised for further testing against data sentences. What Galtung calls traditional science belongs to what above was presented as a science applying LE criteria of validation. For studying civic life and societies such an LE approach functions as a straitjacket preventing the researcher from reaching out to examine values.

Data and theory sentences have descriptive power within what may be called two "dimensions" or two "worlds" of reference - i.e., what is empirically observed and what is theoretically foreseen. What is observed or foreseen is not necessarily preferred (p. 56), so issues of preference (criteria, norms, policy, etc.) cannot be addressed within these two dimensions. One way to break out of this straitjacket is to add value sentences. By introducing these, a third dimension, of preferred worlds, is added to the earlier two dimensions. By introducing values one establishes a basis for addressing what is preferred in addition to that which is observed and that which is theoretically foreseen.

Moreover, a preferred world situation currently may not exist. To adherents of traditional empirical science possible worlds coincided with the empirically known reality. Applied to the fields of social science this leads to theories of a stable status quo or change following known laws of change. Galtung's trilateral form of science presumes a potential re- 
ality broader than known empirical reality. Moreover, theories have to be open-ended. Further, invariances in social science contexts have to be approached (and accounted for) differently than invariances found in natural sciences (p. 68-71).

Galtung (1977) devotes a chapter to what he calls "Science as Invariance-seeking and Invariance-breaking Activity". A proposition is a statement relating a number of variables. It has to be based on a valid data sentence and a valid theory sentence. To become complete the conditions under which it holds have to be specified. If it holds under changes in other variables than those included in the relation, it is said to be invariant under those variables.

The inclusion of a value dimension in science provides the basis for distinguishing two attitudes to invariances. The difference between these two attitudes and their scope of application is best illustrated by a quotation:

... For any invariance is here [in the context of social science studies] considered as an ideological statement, however much it is produced under the banner of value-free, "objective" science. ... A sentence, whether based on data sentences or theory sentences, or both, excludes something. A value sentence, whether based on a goal or an interest, includes something, that which is preferred. As long as what is preferred is also observed and/or foreseen by data or theory, there is no problem. However, the moment what is excluded by data and/or theory is preferred, there is a problem. To raise a confirmed theory sentence, the proposition, of that kind to the level of an invariance is tantamount to saying that something preferred is unattainable. This is vastly different from saying that it was never attained in the past. The latter is only a statement about empirical reality, that which is or was; the former a statement about potentialreality, that which might be — saying that it coincides with what was. In other words, that potential reality coincides with empirical reality - that only what already $i$, is possible, now and in the future.

This becomes more dramatic as soon as different groups in society hold different values, and "science" excludes what is valued by one, and not what is valued by another. In that case, "science" is obviously on the side of the one and not of the 
other, pronouncing as factual what one group wants and as non-factual ... what is valued by the other group. (Galtung, 1977, pp. 73-74. Emphases in original. Text in square brackets added by the author of this paper.)

Galtung (1977, p. 73) perceives a prediction as a specification of an invariance, and an invariance as a generalization involving the future. Invariance seeking research attempts to find general laws on which technologies can be built. Finding invariances within the real $\mathrm{m}$ of natural sciences has stimulated considerable technical development. These kinds of research have mainly been performed by researchers adhering to LE schools of metascience. When research in social and cultural sciences finds apparent invariances Galtung considers them as ideological statements (p. 73). As he briefly states, there do not exist any laws in social science (p. 72). Apparent invariances found in social studies could stimulate further investigations in order to envision possible future worlds in which they are broken. Such studies focus on hidden ideologies and question them. Working dialectically within a school of HD metascience supports invariance breaking activities.

\section{Studying and Meeting Demands for Changing Informing Systems}

New technical artifacts purported to improve informing systems appear on the market at a rapid rate. Their introduction and sales are often boosted by buzzwords. Choosing an appropriate mix of old and new artifacts to inform clientele constitutes a complex task. It has to be built on more solid concepts than current buzzwords.

Also the net of concepts with which we, as researchers, describe the use and evolution of informing systems has to remain open to revision. However, we might avoid some cases of reinventing the wheel if we familiarize ourselves with the longstanding debates about fundamental concepts among philosophers of science. Some aspects of these debates have been indicated in the two preceding subsections.

In many of the disciplines contributing to informing science, LE traditions have dominated and still do so. In the future the field will need researchers and methods of investigation from both the two main traditions. However, in order to present relatively novel ideas this paper has focused and will focus on HD traditions. These, as mentioned above, perceive theory and practice as mutually related. Phenomenology gen- 
erally focuses on everyday life experience. Hence, it has a lot to offer for understanding the use and demand for change of informing systems. Before becoming able to make effective use of a new artifact introduced in an informing system, people have to know how to do so. In this context I would like to focus on two senses of the verb "to know". These can be characterized simply as schoolbook knowledge and practical knowledge. The former corresponds to what manuals offer and brings the learner to the stage of a novice. The latter is acquired in a number of steps by practical use of an artifact. Dreyfus and Dreyfus (1986, pp. 16-51) describe a five step way to acquire professional expertise.

HD schools and Galtung (1977) stress the importance of values external to science for research. Hence, in projecting an investigation or a program for research, the following question should be asked: Who are those who may potentially act differently based on the findings from this investigation or research? Radnitzky (1970, Vol. II, pp. 4-13) calls answers to this kind of question "research guiding interests". Researchers should employ methods of investigation consistent with their chosen research guiding interest. As long as they do this methods might come from both LE and HD schools. This resolves the often heated debates concerning (e.g.) whether researchers in HD-oriented social sciences could use quantitative methods developed within LE schools.

Scientific knowledge, as mentioned before, is necessarily both specialized and fragmentary. Hence, those who attempt to apply it have to form their own syntheses to accommodate the particular situations at hand. Fruitful syntheses could be facilitated by a generic metaphor, which helps to highlight distinctions from newly encountered disciplines or areas of experience. Highlighting them avoids simply reinterpreting them as some already familiar distinction. In the next section I will present a double helix as such a metaphor.

\section{A Double Helix as a Generic Metaphor}

\section{Presenting Living in Time}

As human beings we want to make sense of our lives and the world we experience. However, we seldom become aware of how and how much our everyday manner of talking mediates a world view. Lakoff and Johnson (1980) discuss how our everyday language mediates a world view by metaphors. They report that they have found: "... metaphor is 
typically viewed as characteristic of language alone, a matter of words rather than thought or action. For this reason most people think they can get along perfectly well without metaphor. We have found, on the contrary, that metaphor is pervasive in everyday life, not just in language but in thought and action. ..." (p. 3). Hence, I will use metaphors when I try to relate how I use, redesign, and understand informing systems. Metaphors highlight some aspects of an event, a process, or a thing. At the same time they hide other aspects.

When reflecting on dynamic processes time plays a central role. Some people perceive time to fly like an arrow, progressing linearly. Others perceive it as a circular flow, as when the seasons of the year and birth, life and death endlessly recur. A helix offers a synthesis of these two views. Figure 1 illustrates a helix as a metaphor for related processes perceived as recurring.

The lower part of Figure 1 shows three related processes as a circle. To remind the reader of their processual character I have designated them by verbs. Checkland (1981, pp. 170-171) has given a similar advice to

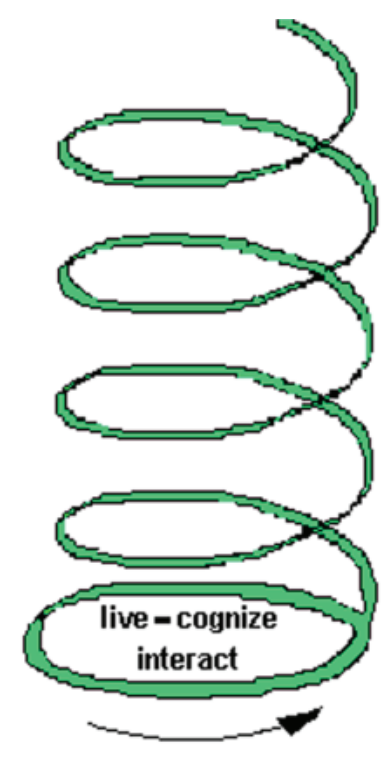

Figure 1. A metaphor presenting related processes as recurring use verbs when referring to human activities.

In lived experience, a person can seldom separate these three processes. Churchman (1971, e.g., pp. 49-56) has discussed difficulties inherent in analyzing and designing systems presupposing separability of their parts. The three processes on which I focus follow research findings by Maturana and Varela (1980). For a number of years, Maturana tried to answer two, what he thought were entirely different, research questions: (a) "What is the organization of the living?" and (b) "What takes place in the phenomenon of perception?" After years of study he arrived at the conclusion that his two questions "...were, in fact, addressed to the same phenomenon: cognition and the opera- 
tion of the living system ... were the same thing" (pp. xii-xvii and p. 13). For this reason I write "live - cognize" in Figure 1.

People interact physically, with each other and with non-living entities. Much of human interaction takes the form of linguistic interaction.

Moreover, "[d]ue to the circular nature of its organization a living system has a self-referring domain of interactions (it is a self-referring system) ..." (p. 10). Maturana and Varela (1980) discuss different kinds of interactions throughout their book (particularly on pages 21-40). Interacting enables us to introduce historical, social, and cultural perspectives. As will be discussed below, each of these three processes entails a number of sub-processes.

People interact with one another both face-to-face and indirectly through different media. People interact not only with their contemporaries, but also interact with people in past and future generations. Finally, they also interact with animals and with all kinds of things and events. Some of these things belong to the set of what we call artifacts. All this remains valid in the upper part of Figure 1, where I metaphorically let the three processes evolve into a helix.

The process I have called "cognize" is comprised of a number of processes - conscious, subconscious, and unconscious - often given other names. Similarly, the processes to live and interact also entail a number of more specific sub-processes. For example, interacting occurs both physically and linguistically, both in and out of face-to-face situations.

Under the word "cognizing" I include such kinds of processes as perceiving, distinguishing, and imagining (cf. Lakoff, 1987, pp. 7-9). More particularly, it subsumes a process generally called "recognize". Often I recognize something as an instance of a category of things or events familiar to me. This presupposes that I have formed a category under which I subsume what I recognize. Those categories which I regard as taken-for-granted, I have formed during my life interacting with others. Many researchers believe that all things or events subsumed under a category share a number of properties and relations. Often, but far from always, this might apply. Lakoff (1987) has developed what he calls a prototype theory of categorizing, in which the traditional view on categories becomes a special case. Here, for example, members of a category share some properties and relations with its prototype. However, they do not necessarily share all of them. An act of cognizing might entail a kind of reframing of something at first recognized as fa- 
miliar. A shift takes place from one frame of reference to another similar to a shift between figure and background. This distinction reminds an observer of the fact that how he reports an observation depends upon the frame of reference he applies.

In cognizing people sometimes become aware of a small domain. At another occasion they might cognize large, even cosmic, domains. Still another time they might cognize many domains of intermediate size. In the course of developing ways in which to present interwoven living, cognizing, and interacting I will, in the next section, distinguish them as two parallel helices.

\section{Distinguishing to Live and to Reflect as Two Domains}

In Figure 2, I present the helix of Figure 1 separated into two helices. As mentioned above, Maturana (Maturana \& Varela, 1980, pp. xii-xvii) has by his research found good reasons to approach cognition and the operation of living systems as the same phenomenon. Still, his earlier position and the position of many researchers in current cognitive science indicate some reason to separate these two phenomena, at least analytically.

From the perspective of Maturana's theories one could consider the two helices in Figure 2 as performed by an observer operating in two distinct domains of reference. One is the domain of immediate experience and the other the domain of abstracted reflection. This explains why the seemingly dualistic presentation in Figure 2 does not violate the (figurative) unity of Maturana's account of both living systems and cognition mentioned earlier.

Another reason for separating the helix of Figure 1 into the two helices of Figure 2 I have found in Schutz (1967). Compared to lived experience conscious reflection, as analyzed by Schutz, always occurs after the event:

... What we, in fact, experience in duration is not a being that is discrete and well-defined but a constant transition from nowthus to a new now-thus. The stream of consciousness by its very nature has not yet been caught up in the net of reflection. Reflection, being a function of the intellect, belongs essentially in the spatiotemporal world of everyday life. The structure of our experience will vary according to whether we surrender 
ourselves to the flow of duration or stop to reflect upon it, trying to classify it into spatiotemporal concepts. (p. 45)

The reader should keep this in mind when studying Figure 2.

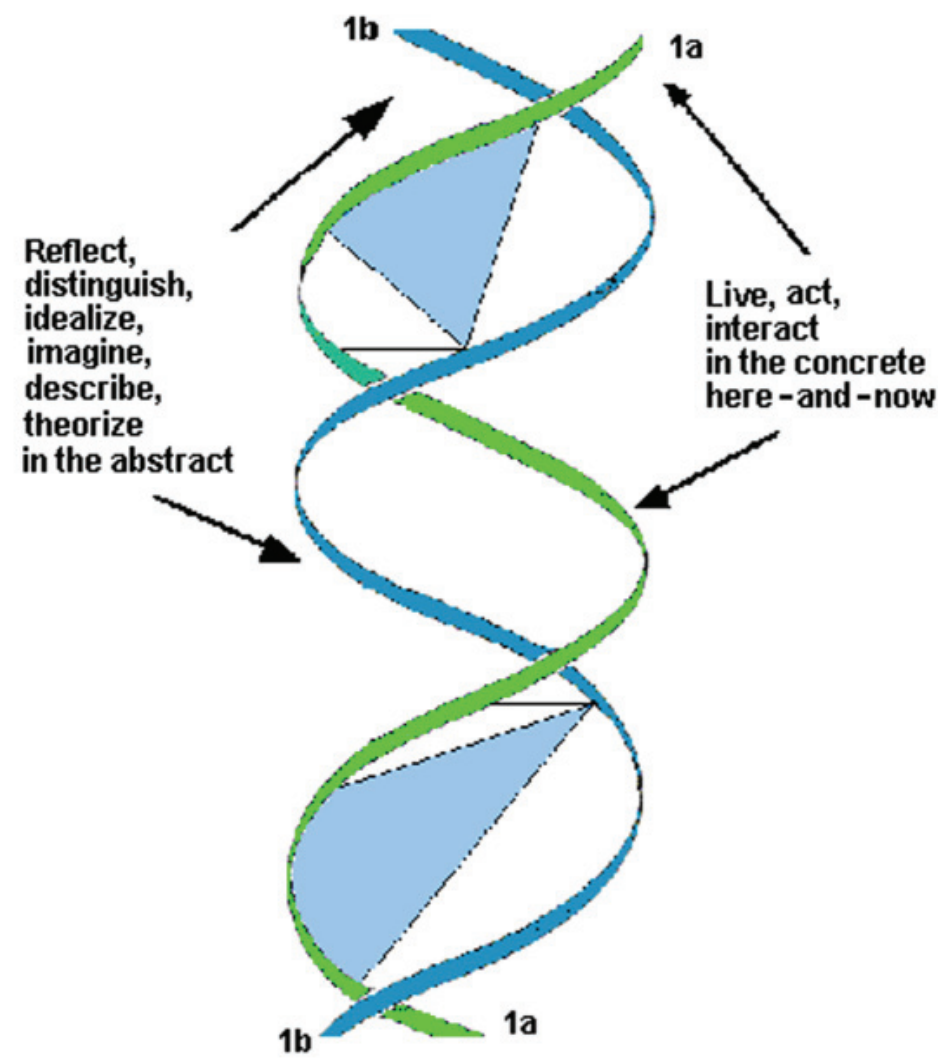

Figure 2. Metaphorically separating living from reflecting

In this form of a double helix metaphor I have chosen to present to "Live, act, interact in the concrete here and now" as one helix. When separating the helix of Figure 1 into two helices cognizing belongs to the domains of both, albeit in different ways. Hence, I do not explicitly use the word "cognize" in Figure 2. The domains of helix 1a entail cognizing on subconscious or unconscious levels. As helix $1 \mathrm{~b}$ I present "Reflect, distinguishing, etc. in the abstract". Cognizing consciously belongs to the domains referred to by helix 1b. My rationale for this 
particular distinction relates to long-standing issues, as explained in the following sections.

\section{Domains referred to by the two helices}

The two helices should help to distinguish two different, although related, sets of domains. "Live, act, interact in the concrete here and now" refers to domains of enacting everyday life as it evolves from situation to situation. This may entail working life situations, but is not limited to such situations. The praxis of everyday life generally entails irreversibly consuming resources.

"Reflect, distinguish, etc. in the abstract" refers to domains of conscious, (potentially) creative activities. Short of enacting some of their results they do notirreversibly consume resources, except the time of people involved. Moreover, when people reflect, both collectively and individually, they catch and express their thoughts using some net of concepts. Mostly, the current net of concepts, in some cultures, helps to make sense of issues reflected upon. When it repeatedly fails to do so it might help to reflect on the net of concepts used. This leads to reflecting on a higher logical level, a meta-level.

Reflecting also plays an important role in designing artifacts and in coordinating their production among lots of different kinds of professionals. For instance, the design and production of buildings, ships and cars furnish examples.

Limitations on available resources make it important to choose which possible ways of life to enact. However, enacting a way of life always has to be done in the face of uncertain and incomplete knowledge.

Moreover, different groups of people will have different value preferences. Of all the new ways of life produced by these creative activities only a few will be enacted. If the process to select which ones to enact retains some altematives, the chances for meeting unforeseen contingencies will increase. So will the chances for supporting the preferred values of different groups of people.

What works in praxis, in some historical time period and within a particular society/culture, can be found by observing the kind of domains referred to by helix 1a. However, it must not be taken as a fact that nothing else could work at some future time or in a different society/culture. This is the message above of Galtung. Nor should it be taken for granted that what works in one society/culture can be trans- 
ferred to another one and will work well there. A number of recent failures in technology transfer between different societies/cultures support this conjecture.

Reflecting activities referred to by helix $1 \mathrm{~b}$ need time and can be supported by stimulating milieus. However, they can evolve and be observed among all kinds of people. They can occur, for shorter and longer time intervals, interleaved among everyday life activities.

Next I will discuss how some features of the two kinds of domains and their relationships are presented by the two helices of Figure 2 .

What the two helices present. Both in research and practice involving use and redesign of informing systems a number of models are used. In research and redesign these models are generally explicit and consciously sharable. In use they are generally implicit and sub-or unconscious. Models present maps of parts of informing systems or what else they purport to present. Explicit models show what their creators have chosen as important to focus on. They belong to domains referred to by helix 1b. These domains entail abstract, idealized, or theoretical images of what people may encounter in domains referred to by helix 1a. In other words I have separated reflecting on what has happened or possibly could happen from living concretely here and now. In the Westem world people seldom remain aware of this distinction. Our reflections only make us partially aware of what has happened or might happen compared with the full complexity of moments lived here and now.

Researchers and systems analysis practitioners use descriptions and models in order to communicate. As reflections of what has happened or might come about, these can only partially or imperfectly portray moments in life experience. This is valid both in the lives of individuals and the lives of groups, communities, and societies.

In Figure 2 I have separated "Reflect, distinguish, etc. in the abstract" from "Live, act, interact in the concrete here and now". As fully awake human beings we always find ourselves situated in an ever moving "here and now". Our technical means of talking and observing over long distances do not change this. However, when we reflect, etc. we, in a sense, can freely move around in space and time. One can apply the metaphor of the two helices both to an individual and to aggregates. This I base on Bateson's (1980) concept of mind which I will present below. (See the section on "Some deeper background ...") The aggre- 
gates may be groups, organizations, societies, or cultures. An inquirer, by choice of a unit of investigation, decides whether individuals or some kind of aggregates are to be focused upon. For a proper understanding of a description or model the presenter should make its unit of investigation explicit. For there to be coherence in a process path from initial analysis through design to development, there must be consistent focus on the same unit of investigation. Moreover, understanding or discussing a description or model has to occur at the proper level of abstraction. Thus I argue, based on Bateson's advice, that one should not mix different logical types when trying to arrive at conclusions.

When a person reflects, her genetic inheritance (phylogeny) and life experience (ontogeny) have formed the preknowledge with which she meets a situation. To discuss and check what she reflects, etc. with others she has to document it in some form. This she might do in a transientor in a permanent form. The need to check what we, as humans, perceive comes from the fact that it might turn out to be an illusion. What we document becomes accessible to others in their domains of personal experience, etc. What thus becomes accessible to others, and ourselves, then can become an object of reflection, etc. Hence, there exist chains of recurring relations between the domains referred to by the two helices distinguished in Figure 2.

Next I will discuss how I perceive the two helices and their corresponding sets of domains as interconnected.

Connecting the two helices and their domains. In the lower part of Figure 2 a sector joins the two helices. This illustrates one way a domain of helix $1 \mathrm{~b}$ may relate to a domain of helix 1a. In a domain referred to by helix $1 \mathrm{~b}$ a group of persons reflect on a past event. When the event occurred it was encountered by people living and acting in a domain referred to by the helix $1 \mathrm{a}$. Some of those reflecting upon the event may have encountered it earlier. The event might have lasted a short or longer time interval as indicated by the time slice shown. Also reflecting, etc. will demand some time interval. The thin horizontal line indicates that this time interval may be of shorter duration than that of the event. This must not become misconstrued as meaning that reflecting can only result in something like mental snapshots. Instead I intend to highlight that when reflecting ex post people's memories of earlier events may have changed. The field of psychology of witnesses furnishes lots of illustrations of this phenomenon. 
In the upper part of Figure 2 another sector joins the two helices. It illustrates another way a domain of helix $1 \mathrm{~b}$ can relate to a domain of helix 1a. This illustrates expecting, predicting, imagining, etc. a possible future event. An expectation might include a short or longer time intervals as indicated by the time slice shown. Also expecting will demand some time interval. The thin horizontal line indicates that this time interval generally tums out as short, compared to that of expected events. Moreover, Figure 2 illustrates the fact that time elapses before the time interval of a prediction or plan occurs. In the mean time unforeseen changes most probably will happen. The aging of requirement specifications for computerized parts of informing systems provides a well known example of this.

In Figure 2 I use the term "idealizing". This refers to the fact that human models always present an idealization of what they purport to show. This is not to say I present the helix "Reflect, etc." as referring to an ideal domain. The helix "Live, act, etc." refers to a domain in which conflicts and unequal distribution of power exist. In fact, the metamodel proposed here will allow researchers and practitioners to consider the role power and conflict have in the social order. Changes reflected upon in the course of living and acting, e.g. in work tasks, will also demand learning by doing. This, however, has to be enacted by people in the domain "Live, act, etc."

These ways of relating the two domains provide a chance to discuss the issue of recurrence mentioned above. Suppose the issue reflected upon is to acquire a package of computer programs purported to improve managerial control of enterprises. Moreover, suppose the reflection ends in a decision to buy and implement the package. The decision entails implementing this package concretely in some part of or the whole enterprise. People in the enterprise in their everyday work will have to learn how to use the package. In essence the process of learning will proceed by trial and error. During the implementation people will start to reflect upon to what extent using the package supports or constrains and complicates their work. These reflections may result in suggestions on how to adapt the package in order to support their work better. To what extent are such suggestions made public? This will depend on how open managers are to suggestions coming from people in lower echelons. Anyhow, suggestions based on lived experience might start off a new round of reflections on feasible and desirable adaptations to the program package. In another paper in this monograph, Kawalek 
reports such a case of problems entailed in transplanting an Enterprise Resource Planning program package from one enterprise culture to a differentone.

This ends my presentation of one way to apply the generic double helix metaphor. I used it to highlight the difference between (a) domains of explicit descriptions, models, plans, theories, etc. and (b) domains in which events evolve and for which the models purport to portray some important aspects. In other contexts a double helix metaphor could be used to highlight other different but linked processes. One case might be to highlight mutual learning betw een workers and designers in redesigning computer applications (Cf. Whitaker, 2007). Observers of some system or systemic entity can observe how its components interact. They can also observe how that entity, seen as a whole, interacts with other entities in its environment. Any relation between the two domains of interaction strictly belongs to the cognitive domain of the observer. As Varela, Thompson, and Rosch (1991, pp. 139-140, cf. above) have argued the nervous systems of living beings work as operationally closed. What happens in the environment of a living system can trigger its nervous system but does not unilaterally control it. The fundamental difference between the two ways of observation can be highlighted by a double helix metaphor. (It falls outside the scope of this paper to show and discuss how this could be done.) However, this paragraph gives a reason why I call the double helix metaphor a generic one.

This ends my presentation of a double helix as a potentially useful metaphor. In what follows I will discuss more important differences between interrelated processes. However, I will not explicitly mark them as potential cases for highlighting by a double helix metaphor. In the next section I will go somewhat deeper in to the background for distinguishing the two domains mentioned above.

\section{Some dee per back ground for distinguis hing two domains}

The Cartesian method, contested by Vico as the only general method of investigation as mentioned above, presupposes a mind-body split. Varela, Thompson and Rosch (1991) offer a resolution of the mindbody problem. Briefly they present their resolution thus:

In fact an important and pervasive shift is beginning to take place in cognitive science under the very influence of its own research. This shift requires that we move from the idea of the world as independent and extrinsic to the idea of aworld as in- 
separable from the structure of these processes of selfmodification. This change in stance does not express a mere philosophical preference; it reflects the necessity of understanding cognitive systems not on the basis of their input and output relationships but by their operational closure. A system that has operational closure is one in which the results of its processes are those processes themselves. The notion of operational closure is thus a way of specifying classes of processes that, in their very operation, turn back upon themselves to form autonomous networks. Such networks do not fall into the class of systems defined by extemal mechanisms of control (heteronomy) but rather into the class of systems defined by internal mechanisms of self-organization (autonomy). The key point is that such systems do not operate by representation. Instead of representing an independent world, they enact a world as a domain of distinctions that is inseparable from the structure embodied by the cognitive system. (pp. 139-140)

By thus characterizing cognition as embodied action they attempt to resolve the mind-body problem and offer, on this basis, an additional system model. For the mind-body problem they offer a resolution by characterizing individual minds as being embodied (1.e., embedded in a given biological system) and interacting in an environment. As mentioned earlier, human beings interact both with contemporaries and with past and coming generations and their cultures. Moreover, they interact with many artifacts and natural objects. By acknowledging and addressing the concept of 'embodiment' as a basis for cognition, this orientation opens the way for accommodating (e.g.) emotions as important factors in human action. This emphasis on embodiment is also reflected in Lakoff and Johnson (1999), who give a philosophical presentation of the ideas about embodied minds, or rather embodied cognition (pp. 94-117).

With regard to system models, Varela, Thompson and Rosch offer a third category augmenting the classic differentiation between open systems on the one hand and closed systems on the other. This is the category of operationally closed systems, which includes (e.g.) the nervous systems of humans and animals and also biological immune systems. Operationally closed systems may exchange material and physically interact with their environment but are not controlled by such exchanges and interactions. This is because the network of their defining proc- 
esses exhibits a mutually integrated closure (in the sense of closure of a directed graph network).

Next I shall address some issues relating to supra-individual (1.e., collective or aggregate) contexts for addressing cognition. Some researchers use terms like mind, memory, and learning to designate collective phenomena, whereas others argue these terms only make sense on an individual level. Bateson (1980, pp. 101-143) offered one way to arrive at a synthesis of these two positions. More precisely, he characterizes minds in a way that can be applied to both individuals and aggregates of individuals.

Bateson's way of characterizing minds is based in part on his key concept of 'difference'. In a section on "Science never proves anything" Bateson first offers a series of seven numbers repeated three times. He then asks the reader to guess the next number. It seems simplest to continue with the first number of the seven number series. He then points out that this guess assumes you can predict the next number based on a rule of simplicity. But as Bateson puts it: "Unfortunately (or perhaps fortunately), it is so that the next fact is never available..." (pp. 35-36). Somewhat later Bateson writes about the impossibility for science to "prove some generalization or even test a single descriptive statement and in that way arrive at final truth" (p. 36).

There are other ways of arguing this impossibility. The argument of this book - which again, surely, can only convince you insofar as what I say fits with what you know and which may be collapsed or totally changed in a few years - presupposes that science is a way of perceiving and making what we may call 'sense' of our percepts. Butperception operates only upon difference. All receipt of information is necessarily a receipt of news of difference, and all perception of difference is limited by threshold. Differences that are too slight or too slowly presented are not perceivable. They are not food for perception.

It follows that what we, as scientists, can perceive is always limited by threshold. That is, what is subliminal will not be grist for our mill. Knowledge at any given moment will be a function of the thresholds of our available means of perception. The invention of the microscope or the telescope or of means of measuring time to the fraction of a nanosecond or weighing quantities of matter to millionths of a gram - all such im- 
proved devices of perception will disclose what was utterly unpredictable from the levels of perception that we could achieve before that discovery. (p. 36-37)

In my current way of expression I would have written "data by means of which we inform ourselves" instead of "information". This, however, does not change Bateson's fundamental argument. In any case, Bateson's construct of 'difference' is the foundation for his explanation of his concept of 'mind'. At the beginning of a chapter on "Criteria of Mental Processes" Bateson (1980) briefly lists six criteria for what he would call a mind:

1. A mind is an aggregate of interacting parts or components.

2. The interaction between parts of mind is triggered by difference, and difference is a nonsubstantial phenomenon not located in space or time; ...

3. Mental process requires collateral energy.

4. Mental process requires circular (or more complex) chains of determination.

5. In mental process, the effects of difference are to be regarded as transforms (i.e., coded versions) of events which preceded them. The rules of such transformation must be comparatively stable (i.e., more stable than the content) but are themselves subject to transformation.

6. The description and classification of these processes of transformation disclose a bierarchy of logical types immanent in the phenomena.

I shall argue that the phenomena which we call thought, evolution, ecology, life, learning and the like occur only in systems that satisfy these criteria. (p. 101-102)

As Bateson (1980) goes on to explain, some aggregates may have parts, which fulfill the criteria above. This could be illustrated by a group of people learning on the group level. The processes of leaming at the group level and at the individual level belong to different logical types. Applying Lakoff's (1987) prototype theory of categorizing learning on the two levels will show different characteristics. Bateson (e.g. on pp. 204210) argues for keeping different logical types clearly separated in descriptions and analyses. Conclusions about phenomena on one level of abstraction based upon data within a different level lack validity. (His detailed arguments for this fall outside the scope of this paper.) 
I shall close with a few comments on Bateson's criteria above. First, he writes about differences as "a nonsubstantial phenomenon" not located in space or time. On the surface this might seem to contradict my assertion that I do not believe in unembodied objects. The noun "difference", in criterion 2 above, does not, however, designate such a thing. One clue lies in the expression that "interactions between parts of mind are triggered by a difference". Another clue appears in criterion 5 . There Bateson states that the effects of difference should be regarded as transforms of events which preceded them. A bell which rings or an expected letter which does not arrive can trigger activities in a person. Bateson (1980, p. 37) points out that there exist thresholds for various differences. If a difference remains below such a threshold it will not become perceived. By referring to human perception Bateson indicates he does not split body from mind.

Bateson mentions "coding" in criterion 5. Coding in his sense is a transform of the effect of a difference. When a person touches a hot surface he almost immediately retracts from it. The heat difference has triggered a train of coded transforms in his nervous system. These result in his retracting his hand from the hot surface. This gives an example of Bateson's sense of coding.

Bateson mentions circular or more complex chains of determination in criterion 4. This corresponds to the operational closure of cognitive systems discussed by Varela et al. (1991). Bateson (1980, p. 141) writes that from his set of criteria it follows that minds have the potentiality of autonomy. This resonates with Varela's (1979) use of the term "autonomy" in relation to operationally and organizationally closed systems a topic beyond the scope of this discussion.

In the next section I will discuss how some meta-scientific perspectives can support research in informing science.

\section{Informing Science and Metascie ntific Perspectives}

\section{Looking for Fundamental Issues}

People have informed themselves and each other for thousands of years. According to some researchers these complexities have increased through the advent of modern communication and data processing technologies. People communicate predominantly by talking and writ- 
ing. When using their mother tongue people seldom become aware of how many social, cultural, and situational clues are involved. Social and cultural features, perceived in abroad sense, can themselves be seen as situational ones. Particularly when people communicate using computers and computer networks of today most situational clues get stripped off as a result of these channels' relatively impoverished capacities.

The paper has pointed out some difficulties in redesigning and using informing systems. Many of these have their roots in human ways of talking and reasoning. Philosophers, linguists, and other researchers have discussed these for many years. The scope and size of this paper forces me to delimit myself to only a few fundamental issues.

\section{Forms of rationality: An illustrative example from pedagogy}

In a world of conflicting interests it seems difficult to agree upon indicators of effectiveness. A comprehensive discussion of this issue falls outside the scope of this paper. However, I will illustrate the most salient points by reference to a relevant discussion comparing rationalities.

In the late 1970s, Dunne (1993, p. 1), and his colleagues were "formally introduced to a new model of teaching that promised ... spectacular improvements in the quality of our students' teaching if only they (and we as their mentors) would use it as a blueprint in planning and conducting lessons". The model was called the objectives model and opened the road to efficieng in teaching. Dunne, and good fellow teachers he knew, felt that the objectives model ran up against their experience in classrooms. This triggered him to examine closely representative texts on the objectives model. This examination brought him to study a larger philosophical context and to write his book.

Later in his introduction Dunne summarizes his critical views of the objectives model in pedagogy:

... Nor is there any sense that ... something might be at work in the pedagogic situation which cannot simply be made the object of analysis but rather must be lived through - a kind of subsoil which nourishes the fruits of explicit purposes but which is notitself a fruit. It is as if action can be resolved into analysis - that the problems of the first-person agent can be solved from the perspective of the third-person analyst. As a form 
of action, then, teaching is no longer seen as embedded in particular contexts or within cultural, linguistic, religious, or political traditions which may be at work in all kinds of tacit and nuanced ways in teachers and pupils as persons. (p. 5) (Emphases added here.)

Dunne's illustrative background uses an example from teaching, one form of informing people. However, this quotation to me seems, mutatis mutandis, applicable to other similar relations. For instance, there are parallels here to the relations that exist between system analysts and workers. The analysts remain third-person observers. The workers, as first-person agents, have to make sense, in particular situations, of data from data processing systems. To acknowledge this fundamental difference mightimprove mutual understanding and communication between the two parties. Advocates of participatory design (PD), as for instance, Ehn (1988) based the notion of PD on this very point.

Dunne (1993, p. 5-6) describes the logic behind the objectives model as an instrumentalist one. Its adherents saw means in themselves as value neutral and hence substitutable in principle by any other means. All questions of value became located at the level of (or translated into) ends. Value discussions, however, became attenuated by demanding ends which had become cast into discrete, observable behavior. To evaluate atomistic objectives requires study of their effects aggregated over time. The adherents of the objective model did notinclude this kind of evaluation on a higher level in the techniques they recommended. Dunne admits that instrumental reason constitutes a logic. But he does not accept it as a universal standard that should determine all rational action:

... the problem confronting me was to show that this standard [of instrumental reason] constituted $a \operatorname{logic}$ or $a$ form of rationality - one which has its own biases, limitations, and (when these limitations were not acknowledged) distortions - and that it did not, therefore, define exclusively what is meant by 'logical' or coincide with rationality as such. (pp. 7-8) (Emphases in original. Text in square brackets added here.)

Dunne confronts his problem by a number of conversations with the philosophers Aristotle, Newman, Collingwood, Hanna Arendt, Gadamer, and Habermas. The supplementary form of rationality he offers I roughly characterize as a historically, linguistically, and cultur- 
ally based rationality exhibited by people in their everyday action. Dunne states that his critical analysis of forms of rationality applies not only to pedagogy but to other fields too. He mentions political activity, organizational and management practices, psychotherapy, and community development (p. 8). All these fields involve people informing themselves and others. The rationality of instrumental reason corresponds to the view on rationality within LE traditions. (The supplementary form of rationality offered by Dunne corresponds to the view on rationality within $\mathrm{HD}$ traditions.)

\section{Language use and calculi}

Both in creating and in using informing systems we, as human beings, predominantly use language. There are significant difficulties in analyzing and changing habits of language use. From childhood we have become socialized to communicate by talking and writing. Can there possibly exist any difficulties in this? A metaphor might help to highlight the problems of analyzing, understanding, and changing habits of linguistic communication. As Figure 3 indicates we live and interact in language like fish live and interact in water. Similarly as fish live in water we live in languaging. (The Glossary at the end of this monograph gives an explanation of this term borrowed from Maturana, 1988.)

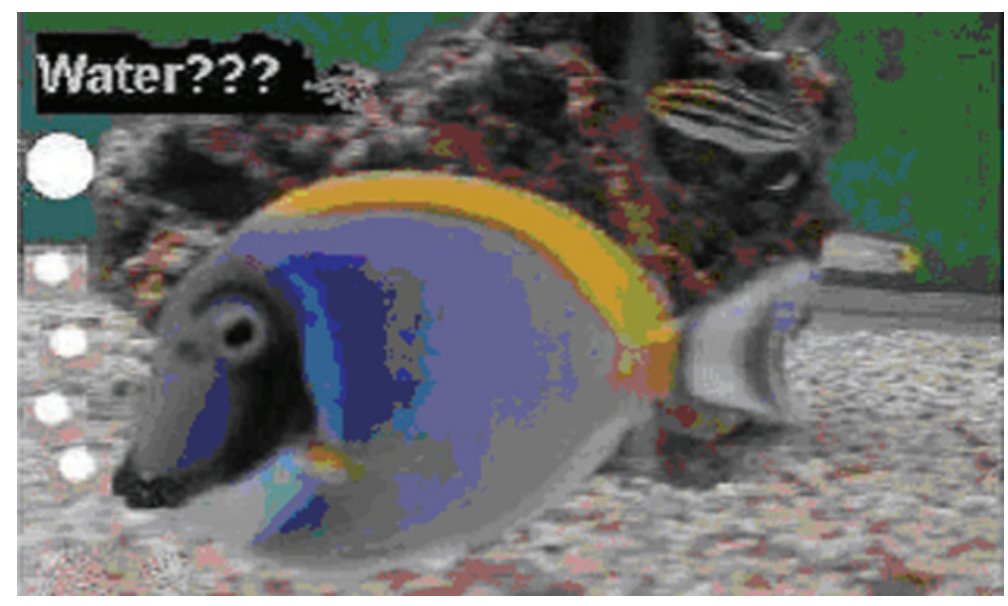

Figure 3. Man in Languaging like fish in water?

In his conversation with Gadamer, Dunne (1993) writes about language and language use in practical situations. Computerized parts of inform- 
ing systems not only transmit linguistic and other symbolic expressions over space and time; they also transform some expressions into new ones. For example, they can be programmed to follow ordinary or matrix algebra. They can also be programmed to draw logical conclusions, generally according to first order predicate logic. This, too, is a form of calculus.

However, to perform calculation as computers do, entirely based on syntactic form, puts severe restrictions on the language used. The philosopher Wittgenstein reflected deeply over different language forms. In Wittgenstein (1974, originally published 1921) the author devised a language suitable for deductions by logical calculation. This becomes clear when Wittgenstein (1974) in his statement 3.33 writes:

In logical syntax the meaning of a sign should never play a rôle. It must be possible to establish logical syntax without mentioning the meaning of a sign: only the description of expressions may be presupposed.

Here the description of an expression refers to the description of its form. By "logical syntax" Wittgenstein refers to rules of transformation of a calculus. In his later writings, for instance, in Wittgenstein (1958, 1963), he stresses that language as used in everyday life does not resemble such a calculus:

... For remember that we in general don't use language according to strict rules - it hasn't been taught us by means of strict rules, either. $W e$, in our discussions on the other hand, constantly compare language with a calculus proceeding according to strict rules.

This is a very one-sided way of looking at language. In practice we very rarely use language as such a calculus. For not only do we not think of the rules of usage - of definitions, etc. while using language, but when we are asked to give such rules, in most cases we aren't able to do so. We are unable clearly to circumscribe the concepts we use; not because we don't know their real definition, but because there is no real 'definition' to them. To suppose there must be would be like supposing that whenever children play with a ball they play a game according to strict rules. (Wittgenstein, 1958, p. 25) 
This quotation illuminates, mutatis mutandis, why workers encounter difficulties explaining everything they need to know in order to perform a task. This depends on the specific circumstances in particular work situations. Still, system analysts generally ask workers to describe their work precisely as part of requirements engineering.

As a metaphor for language use in practical life Wittgenstein (1963) introduces what he calls "language games". By means of these he puts language use into the stream of everyday life situations. Here I will take advantage of the way in which Blair (2006) explains Wittgenstein's metaphor of language games:

The language-game is one of the most important components of Wittgenstein's philosophy of language. Wittgenstein resists, as we might expect, giving a rigorous definition of it, but that does not mean that it is not a rigorous notion. As usual, we must see its rigor in the examples Wittgenstein gives us. The problem that Wittgenstein faced was how to reconcile the need in language for a predictable structure that determines how words in language go together, with the simultaneous need for flexibility in usage. Further, the structure must be dynamic: it must be able to account for regularities in language that extend over time (such as holding a discussion). Structure in language is necessary so that we can speak in predictable ways and be understood, but flexibility is necessary, too, in order to permit new or creative uses of language - something important when we face new or different situations, or have to describe something we don't understand well. These situations do not have to be entirely new, but merely new to the speaker. The notion of a language-game provides both the predictable structure in language as well the flexibility that allows us to talk about new or unusual situations. Consider the game of baseball, for example: there are rules, that can be codified and written down, but within these rules there is enormous latitude for innovative play... (p. 80)

Blair (2006) then goes on to make a list of the important aspects for language games. He adds that this list is not meant to be regarded as exhaustive or final. Nor is it necessary that all games exhibit all these aspects. A brief presentation of the list follows: 
1. A predictable structure: Usually codified as a set of rules and may be written down in some sports, like baseball or football, or may be proposed informally by casual players of a game, such as two children playing catch. ...

2. A point or a goal: This provides a focus for the intentions of the "players", al though not all games, as Wittgenstein rightly points out, have a goal ...

3. Flexibility of performance. There is a wide latitude in terms of what kind of performance is permitted within the boundaries of the game's structure. ...

4. The need for training and practice: In order to "play" the game, one needs to be taught how to play. Some of this teaching may take the form of explanation, but most of it takes the form of being coached - trying to play the game and have a coach offer suggestions. ...

5. Performance is not necessarily accompanied by conscious mental processes: Many highly skilled athletes claim that they have little conscious thought during their performance. ...

6. The ability to formulate families of games - to derive new games out of old ones: Many games grow out of existing games and derive their rules and intent from previous games. One needs only to think of American baseball and its antecedent game of English cricket. ...

7. Games can rely on other games for their codification....

8. Games are imbedded in, and influenced by the larger context of human activities. This provides a way of instilling extraordinary complexity into a game without having to codify all of the complexity ...

9. Games help individuals build and refine their social and interpersonal skills, such as, the ability to follow rules reliably, the ability to make and interpret rules, the ability to coordinate one's actions with others, etc.

10. Games take place over time, ... (pp. 80-84)

I have included this lengthy excerpt from Blair (2006) for two purposes. It sheds light upon Wittgenstein's concept of language games'. It also suggests a potentially useful similar concept of 'work-task-games'. 
Workers have difficulties in describing their work tasks precisely to designers of computer support. Moreover, they need to learn and acquire skills in the modified work-task-game to play, once some new computer support has become implemented. It falls outside the scope of this paper to develop this suggestion further. Nevertheless Wittgenstein offers developers some alternative ways to investigate work tasks.

\section{Inform ing systems}

Generalized informing systems. In the introduction I generalized the concepts of 'informing science' and 'informing systems'. In the mean time I have introduced Bateson's generalized concept of mind. As one of his criteria for minds he mentions circular (or more complex) chains of determination. These I call "closed cause-effect chains". Bateson also argued that to understand processes of learning it would help to look for such chains. To learn means to know more, individually or collectively. To inform oneself or others implies an intention to make people more knowledgeable. By her autonomy of interpretation an orientee has to safeguard herself against not well supported messages and misinformation. Informing will be better understood if studied as comprising circular and more complex closed chains of determination. I put this forward as a conjecture of my own.

On this conjecture I based my way to delimit informing systems to include parts of closed cause-effect chains that are not confined to artifacts. In some subsystems of informing systems, however, closed cause-effect chains are confined to artifacts, for instance, systems dedicated to support automatic control of machinery. Systems controlling ignition in cars, or industrial robots, or executing programs of washing machines offer examples of the latter kind of systems. (They might be called "automatic control systems" rather than "information systems".) Many more such systems, embedded to control functions of machinery, will be designed over the years to come. For such systems to work reliably the interpretation of data has to follow formal rules based on two-valued logic. The processes in what I have delimited as informing systems operate at a logical level of a different type. Suffice it to indicate that these processes must accommodate Hegelian dialectics. In other words such processes must be able to handle a thesis, its antithesis, and the resolution of their apparent contradiction by an innovative synthesis on a broader level of abstraction. 
In what follows I write about informing systems in the sense explicated. Systems supporting automatic control of machinery should rather be called "automatic control" or "data processing" systems. In the next section I will briefly sketch the history of the term "information system" used so far. Informing systems including computers as parts will form the main subject area for informing science. How ever, according to my view, informing systems do not need to entail computers. Otherwise, all comparisons with how people informed themselves and each other before the event of digital computers would be excluded.

Describing informing systems. Next I will focus on two recurrent sequences of processes which constitute creating and using informing systems. As an area for academic studies information systems appeared with the advent of computers in the middle of the 20th century. How to define the term "information system" still seems open to debate. Historically, when computers were offered to support business tasks, vendors offered them as performing "electronic data processing". After a number of years vendors offered computers as capable of performing "information processing". Lately computers have been offered as performing "knowledge processing". During the last 60 years the amount of data a computer can handle and the speed in handling them has increased dramatically. This has certainly brought a great number of new applications into the realm of (digital) data processing. Has the success of von Neumann computers warranted perceiving them as equivalent to all kinds of human information or knowledge processing? (The majority of computers in use today are built according to a von Neumann architecture. For a brief explanation of a von Neumann computer, see the Glossary at the end of this monograph.)

My answer to this question is: No! The reasons for this answer can be briefly summarized. To perform processing a computer needs a program. A computer program directs a computer to operate strictly according to the form of its input data or of some data in its data base. It may also operate according to the form of data internally stored earlier during operation of a program. Finally, as a result of its operation it presents some output data. Here I limit my discussion to cases in which people have to interpret these data, i.e. in agreement with my delimitation above of informing systems. Based on the outcomes or outputs system users decide which action to take, if any. System analysts and programmers have in designing a program assigned standard interpretations to different patterns of data. By these schematic interpretations 
they construct a schematic form of a 'language'. Such a concept of 'language' enables users of an application program to furnish input data and to make sense of output data. This, however, is valid only in relation to some limited domain of application. To keep track of the events in an inventory of spare parts for a number of car models illustrates one such domain of application. To keep inventory records for many thousands of articles manually constitutes a task that demands work by many people. No doubt a computer program can support this kind of task.

However, the expressiveness of schematized 'languages', which a computer can process according to their form, is very limited compared to that of natural languages. The reason is that contexts given in standard interpretations of programs are very limited. The ways in which situational contexts offer cues to interpretation in human communication are more complex and dynamic. A deeper analysis of the limits of schematic languages, basic to computer programs, can be found in Whitaker (1992, pp. 42-50). The schematisms used, moreover, exclusively build on set-theoretically based categories.

These exclude all types of Lakoff's (1987) prototype categories. This, hopefully, indicates that output from computer processing may better be looked upon as data in need of human interpretation before being acted upon. Such a view helps in assessing information system performance with a critical attitude in light of the live situation of application.

Lately the field of information systems has broadened to subsume also journalism and education. Hence it seems appropriate to talk about informing systems rather than information systems. This amplifies the need to distinguish informing systems in the sense explicated above. Such a shift in terminology offers four advantages. First, it characterizes the informing system as something people create to inform themselves and others. Second, people have done so for thousands of years. This opens a way for historical comparisons and a consideration of the role that social and cultural context plays here. Third, it also allows for people interpreting data they gather or receive before reorienting themselves. However, the orientee's reorientation might differ from the one the orienter intended to achieve. Fourth, changing informing systems today will generally entail taking advantage of new affordances offered by communication and computer technologies. The term "information system", coined in parallel with computers, plays down the role of peo- 
ple involved in their use. IS experts, as designers, and company experts consulted during requirements specification become the people who count, relative to those people who will actually use the resultant computer support. The term "informing systems" opens up a possibility for studying people who use them at least as much as those involved in their design. Moreover, in their work and life situations people should be studied as professionals in their own work and life.

This brings me to how people become involved in the recurrent processes of use and redesign of informing systems.

Recurrent processes of informing systems use and redesign. In Figure 4 I present, in a simplified way, some recurrent processes in informing systems use and redesign. In it I distinguish two different flows of human communication. The outer flows I have called "Face-to-face and other communication not manipulated by computers". The inner flows I have called: "Computer manipulated communication". The words "outer" and "inner" I use only to refer to them in Figure 4. An important difference exists between these two flows of communication. The expressiveness of what can be mediated by the outer flows is an order of magnitude larger than what can be mediated by the inner flows. This is particularly true when it comes to flows that are manipulated by computers to reach conclusions by applying two-valued logic. In the outer flows the often perceived gap between intellectual thoughts and emotions can be bridged. Artists and poets are those people who often best bridge this gap (See Bateson, 1972, pp. 448-465).

Figure 4 intends to underline the fundamental non-separability of for$\mathrm{mal}$, in the sense of based on explicit rules, and non-formal communication, guided by evolving Wittgensteinian language games. Both in specifying rules for formal communication and in learning how to interpret and act upon messages from formal systems non-formal communication will always be needed.

Hence, studies of informing systems cannot entirely ignore non-formal communication. This highlights one reason to approach the field of informing science as a transdisciplinary one.

Human communication I interpret in a very broad sense. Following Watzlawick, Beavin, and Jackson (1967) I take it that people communicate by all kinds of behavior. In a section on "The Impossibility of Not Communicating" they write: 


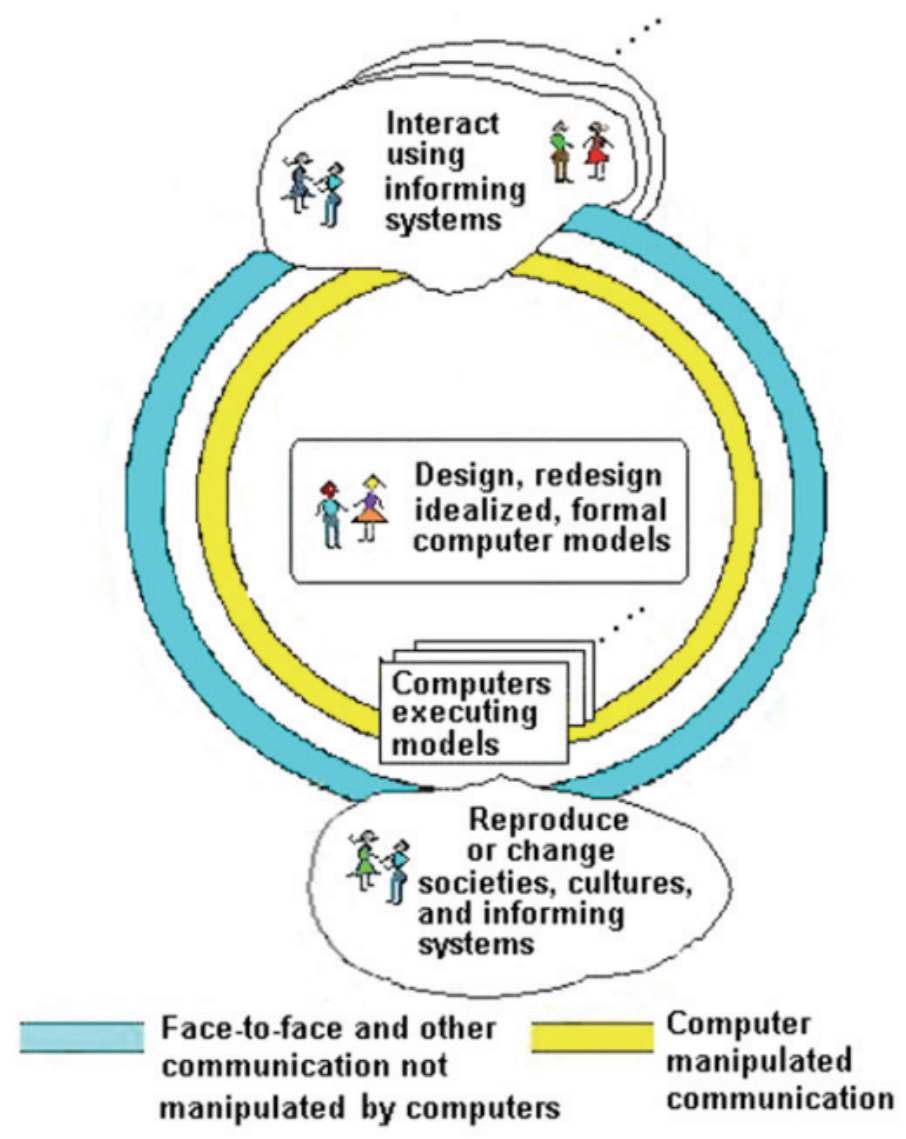

Figure 4. Recurrent processes of informing systems use and design

First of all, there is a property of behavior that could hardly be more basic and is, therefore, often overlooked: behavior has no opposite. In other words, there is no such thing as nonbehavior or, to put it even more simply: one cannot not behave.

Now, if it is accepted that all behavior in an interactional situation has message value, i.e., is communication, it follows that no matter how one may try, one cannot not communicate. Activity or inactivity, words or silence all have message value: they influence others, and these others, in tum, cannot not respond 
to these communications and are thus themselves communicating. ... (pp. 48-49)

Moreover, according to Watzlawick, Beavin, and Jackson (1967) there exists both a content and a relationship level of communication:

... in the foregoing it was suggested that any communication implies a commitment and thereby defines the relationship. This is another way of saying that a communication not only conveys information, but that at the same time it imposes behavior. Following Bateson ... these two operations have come to be known as the "report" and the "command" aspects, respectively, of any communication. ...

The report aspect of a message conveys information and is, therefore, synonymous in human communication with the content of the message. It may be about any thing that is communicable regardless of whether the particular information is true or false, valid, invalid or undecidable. The command aspect on the other hand, refers to what sort of a message it is to be taken as, and, therefore, ultimately to the relationship between the communicants.... (pp. 51-52)

As indicated at the top of Figure 4 there exist many situations in which people interact. This goes for work situations as well as for situations in everyday life. In all of these, people inform themselves or become informed by others by data from a number of informing systems. Data from different sources sometimes suggest choosing (and enacting) different ways of action. In such cases experience of the extent to which a data source has proved reliable earlier comes into play.

Some data come from the execution of idealized, formal models on computers. A new source of such data first has to earn trust by showing its reliability. Formal models executed on computers have become more complex. This generally makes it difficult to understand such models and to evaluate the data they furnish. So far requirements engineering has loomed large in research and education with its focus on computerized parts of informing systems. However, idealized, formal models never could or should cover all situations workers meet. Hence, the workers and their managers need to understand the limits of applicability of the computerized models. This goes far beyond the introduction, training, and on line help now given when introducing a new computer application. 
Computer models only offer sensible advice for a part of the class of situations they have been designed to cover. Moreover, the scope of their successful application diminishes with time. The answer to this generally is that new, more advanced models will be developed. However, first-person actors still have to handle all situations occurring in the mean time. Given a fair amount of autonomy and license to use it, they can effectively handle situations outside the scope of the current computer model. This, however, also presumes that they recognize, within their frame of reference, the strengths and limitations of the model.

Recall the discussion above about the limitations of the schematized 'languages' intended to explain models embedded in computer programs. Recall also the rather common, but mistaken, belief that computers handle information and knowledge the way people do. A computer program cannot go outside of the restrictions and values embedded in it. Generally computerized models evaluate situations only along one value scale. Often this is a money scale according to prices estimated as valid in some limited time period. People, however, seem capable of shifting between different mental models depending upon how a situation develops. Admittedly, some mental models might be outdated and some simply wrong. The fact that people make mistakes is often taken as a strong reason to let a computer choose the right decision. Such a conclusion misses an important point. It is not possible to predict the right decision for all possible situations including how to evaluate them and the outcome of a decision. Moreover, the more types of situations a program has to handle the more complex it becomes.

A majority of cases a computer may decide automatically based on a set of rules and values specified in advance. These, the workers responsible for handling the cases can devote a small amount of their time and effort to monitor. A minority of cases, selected according to criteria given by the workers, could be brought to the workers' attention and handled by them. These criteria of selection the workers should be enabled to adjust. In a sense these cases could be called exceptional cases. How to handle them is part of the policy of the enterprise. Hence both the workers and their managers can be involved in handling them. Moreover, they are particularly sensitive to the situation in which they occur. Interactive programs have been technically feasible for quite some time. Program designers might now relax the traditional idea of trying to ap- 
proach $100 \%$ automation of cases turning up during requirements engineering. Instead they could focus on a flexible interaction between simple, understandable programs and the workers using them to handle their tasks.

The model or set of models a program implements and its variety remains until the program is redesigned. The variety of concrete situations to be handled according to what the computer model suggests increases with time. The additional variety in actions to handle them adequately has to be generated by human actors. They may learn this the hard way by trial and error. If these actors understood the models embedded in programs and their limitations they would be able to decide more confidently when and how to use their full action repertoire. At least that is a conjecture of mine. To achieve this, the models embedded in the programs should be made visible (with respect to presence and coverage) and transparent (with respect to structure and implications).

To this end it has to be made clear which action alternatives a model generates and how it evaluates them. Moreover, it has to be stressed that an embedded model cannot generate other types of action alternatives or evaluate them in other ways. In order to bring this home it will help to look upon what computers do as data processing. As mentioned earlier, data processing differs from information or knowledge processing, whatever these terms might refer to. Computer program embedded models have to be made transparent by explaining them in the language of their users. Such explanations offer what I will call model transparency.

At least for some computer programs producing and making such explanations assessable should be part of program design. Many programs in which data output guides human action belong to this category. To produce these explanations during program design I will call model transparency engineering. Today use cases, in some form, are collected during requirements engineering. These form a basis upon which models are constructed. They become embedded in programs in the form of models. The majority of those who will be supported by a computer program do not know anything about these use cases. To present them and the fact that the computer will treat all cases as one of these types is part of model transparency engineering. How to do this and what more may be needed lies outside the scope of this paper. 
Some aspects of the model transparency problem I have found discussed in Dinka (2006, chapters 7 and 8). Dinka studied a 3D visualization tool offered in a program intended to support Gamma knife brain surgery. He found that the professionals planning the surgery did not use the 3D visualization. They relied instead on sets of $2 \mathrm{D}$ magnetic resonance images. Briefly, it was found that the designers had built a 3D functionality which was not important to the professional users. The functionality did not fit the professional experience of neurosurgeons and physicists involved in the planning. Above, a distinction made by Dunne (1993) between first-person and third-person perspectives was mentioned. Dinka's study illustrates the risks of designing from a designer's perspective and not from that of a professional user. There also might exist lessons to be leamed from simulation programs for training pilots, surgeons, and business managers.

So far I have only mentioned that people interact with computers as one way of informing them selves or others. However, they also furnish a lot of data that gets processed by computers. If no computer application returns support valuable to these people the situation entails a risk of low data quality.

Figure 4 also indicates how societies, cultures and un-computerized parts of informing systems reproduce or change. Within societies and cultures there exist a number of sub-communities. To simplify Figure 4 I have not explicity indicated this. These processes differ from the large part of interactions in everyday life. Most everyday interactions people perform to achieve some kind of intended goals, or at least something we can interpret as a goal. By the very way in which they do this they mostly reproduce existing societies, cultures, and informing systems. Most people will rarely - if ever - notice effects of their dayto-day interactions in stabilizing or preserving the status quo. In stabilizing societies, cultures, etc. both non-computer manipulated and computer manipulated communication play a role. In the course of changing societies, cultures, etc. face-to-face and other informal forms of communication play the by far dominant role. Among these informal ways of communication I include use of cell phones, bulletin board systems, and blogs. As I see it, these do not represent idealized, formal models executed on a computer.

To summarize, Figure 4 illustrates the following points. Many informing systems today involve some computer supported data processing. However, a responsible human actor using such a system always needs 
access to other channels of communication too. Two suggestions can emerge. (1) Be selective in choosing which use cases, turning up during requirements engineering, to implement in a particular application program. Resist the professional temptation to cover all. Unforeseen cases may always be expected to turn up soon. (2) Two computerized models implementing different value criteria might inform a human actor better than one. This means studies of use could profit from focusing on more than computerized parts of informing systems alone. Studies of what workers or other people using computer applications actually do will reveal such additional aspects of the use context. Researchers from disciplines other than information and computer science will probably help us understand this more easily. The figure also indicated the need to focus on a new area to support effective use: model transparency engineering.

\section{Conclusions}

Informing science studies need to draw on traditions of inquiry from several meta-scientific schools. In reporting studies and findings authors should always make explicit whose interests have guided the inquiry. Moreover, they also should make known to what extent the interests of other stakeholders involved have been used as restrictions. Methods of investigation should be used in ways which support the research guiding interest. If used according to a specifiable research guiding interest, it does not matter within which tradition they were first developed.

Researchers and practitioners in informing science have very different backgrounds, training, and experience. This creates a problem of communication in collaboration with colleagues from other disciplines. Familiarity with metascience could help in this communication by furnishing all participants a kind of meta-language in which to discuss across disciplinary borders.

The generic metaphor of a double helix can be used to highlight important distinctions. In this paper it has illustrated the distinction between describing and reflecting and living in a complex here and now. A few more distinctions, which could be highlighted by a double helix metaphor, have been indicated.

Both informal and formal channels of communication are needed for people to inform themselves and others. These should be perceived as 
supplementing each other. By most everyday communication people reproduce the societies and cultures in which they live, generally without being aware of this. In stabilizing societies, cultures, etc. both noncomputer manipulated and computer manipulated communication play a role, whereas face-to-face and other informal forms of communication play the dominant role in changing societies, cultures, etc.

Finally, discussions of how to improve computerized parts of informing systems have to date focused on how to improve requirements engineering. The use of these parts of informing systems in the future could more effectively support clientele by developing and implementing methods for model transparency engineering.

\section{Acknowle dge ments}

The author is grateful for the insightful comments of the reviewers. Moreover, I like to thank Professor James Nyce and Dr. Randall Whitaker for their extensive study and valuable comments to earlier drafts of this paper. Finally, I want also to thank my co-editor, Christine Welch, for a careful reading of the paper and a number of suggestions to improve its clarity and English. However, it is I alone who should take full responsibility for the content.

\section{References}

Bateson, G. (1972). Steps to an ecology of mind: A revolutionary approach to man's understanding of bimself. New York: Ballentine Books.

Bateson, G. (1980). Mind and nature. A necessary unity. London: Fontana Paperbacks. (First published by Wildw ood House in 1979.)

Blair, D. (2006). Wittgenstein, language and information: Back to the rough ground. Dordrecht: Springer.

Checkland, P. (1981). Systems thinking, systems practice. Chichester: John Wiley.

Churchman, C. W. (1971). The design of inquiring systems. New York: Basic Books.

Cohen, E. (1999). Reconceptualizing information systems as a field of the transdiscipline in forming science: From ugly duckling to sw an. Joumal of Computing and Information Technology, 7(3), 213-219.

Dinka, D. (2006). Role, identity and work: Extending the design and development agenda. (Doctoral dissertation, Linkoping University, Sw eden. Linkoping 
Studies in Science and Technology No. 1008.). Retrieved November 25 from http://www.dinka.se/Dinka/Research_files/PhD.pdf

Dreyfus, H.L. \& Dreyfus, S.E. (1980). Mind over mach ine: The power ofhuman intuition and expersise in the era of the computer. Oxford, UK: Basil Blackw ell.

Dunne, J. (1993). Back to the rough ground: 'phronesis' and 'techne' in modem philosophy and in Aristotle. Notre Dame, IN: Indiana University of Notre Dame Press.

Ehn, P. (1988). Work-oriented design of computer artifacts. Stockholm:

Arbetslivscentrum. Diss.

Galtung, J. (1977). Essays in methodology. Vol. 1. Methodology and ideology. Copenhagen: Ejlers.

Giddens, A. (1984). The constitution of society. Cambridge: Polity Press.

Kaw alek, J. (2007). Pedagogy and process in "organisational problem-solving". Informing Science, 10. [In this monograph.]

Lakoff, G. (1987). Women, fire, and dangerous things: What categories reveal about the mind. Chicago: The University of Chicago Press.

Lakoff, G., \& Johnson, M. (1980). Metaphors we live by. Chicago: The University of Chicago Press.

Lakoff, G. \& Johnson, M. (1999). Philosophy in the flesh: The embodied mind and its challenge to western thought. New York: Basic Books

Maturana, H. R. (1988). Ontology of observing: The biological foundations of self consciousness and the physical domain of existence. In R. Donaldson (Ed.), Conference w orkbook Texts in cybernetic theory: An in-depth exploration of the thought of Humberto Maturana, William T. Powers, and Emst von Glasersfeld". American Association for Cybernetics, 1988. Retrieved November 4, 2006, from http://www .inteco.cl/biologv/ontology/

Maturana, H. R. \& Varela, F. J. (1980). Autopoiesis and cognition: The realization of the living. Dordrecht: D. Riedel. (First published in Chile 1972 under the title De Machinas y Seres Vivos.)

Radnitzky, G. (1970). Contemporary sch ools of metascience (Second revised edition). New York: Humanities Press and Gothenburg, Sw eden: Akademiforlaget.

Schutz, A. (1967). The phenomenology of the social world. (G. Walsh \& F. Lehnert, Trans.). Evanston, IL.: Northw estern University Press. (First published in German 1932.)

Stanford encyclopedia of philosophy. (2007). Giambattista Vico. Retrieved from http://plato.stanford.edu/archives/spr2007/entries/vico/

Varela, F. (1979). Principles of biological autonomy. New York: Elsevier. 
Varela, F. J., Thompson, E., \& Rosch, E. (1991). The embodied mind: Cognitive science andhuman experience. Cambridge, MA.: MIT Press..

Watzlaw ick, P., Beavin, J., \& Jackson, D. D. (1967). Pragmatics of human communication. New York: Norton.

Whitaker, R. (1992). Venues for contexture: $A$ critical an alysis and en active reformulation of group decision support systems. Umeå (Sw eden): Umeå Universitet, ADB (Dept. of Administrative Data Processing / Informatics) dissertation / report UMADP-RRIPCS 15.92 .

Whitaker, R. (2007). Applying phenomenology and hermeneutics in IS design: A report on field experiences. Informing Science, 10. [In this monograph.]

Wittgenstein, L. (1958). The blue and brown books. Oxford: Basil Blackw ell.

Wittgenstein, L. (1963). Philosophical investigations. (G.E.M. Anscombe, Trans.). Oxford: Basil Blackw ell. (First edition 1953.)

Wittgenstein, L. (1974). Tractatus logico-philosophicus. German-English text. (D.F. Pears \& B.F. McGuinness, Trans.). London: Routledge \& Kegan Paul. (First German edition in Annalen der Naturphilosophie, 1921.)

\section{Biography}

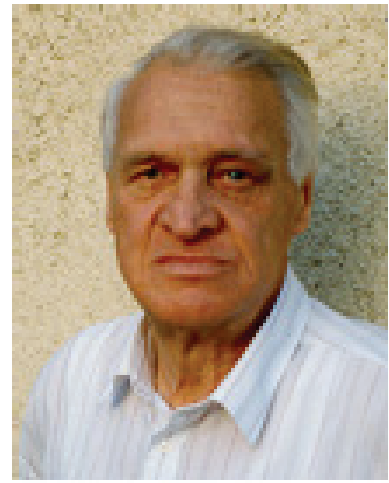

Professor Emeritus Hans-Erik Nissen has served since 1991 as a senior research fellow at the department of Informatics at Lund University after years of demonstrated research excellence within the information systems field. He graduated in chemistry from the Royal Institute of Technology, Stockholm. He then for many years worked in various positions in the Swedish Pulp, Paper and Timber industry before reentering academia. The seven last years of these he started and headed the first computer

center of the Swedish Cellulose Company (SCA). 